\title{
Responsabilidad y evidencia en la Crisis
}

\author{
Vania Alarcón \\ Pontificia Universidad Católica del Perú
}

Resumen:

El presente texto vincula el concepto de responsabilidad descrito por Husserl en la Crisis al tercer sentido de evidencia, correspondiente, a su vez, al tercer momento de la delimitación de lo inmanente y lo trascendente. En la primera parte del trabajo se revisa el concepto de evidencia; en la segunda, el de intencionalidad; $y$, en la tercera, el de responsabilidad, para lo cual se esbozan aquellos de la libertad, la idea de la humanidad, la historia y la tarea de la filosofía.

Palabras clave:

Husserl, fenomenología, responsabilidad, evidencia, Crisis

\begin{abstract}
:
This text links the concept of responsibility described by Husserl in the Crisis and the third meaning of evidence, which corresponds to the third moment of the delimitation between what is immanent and transcendent. First, the concept of evidence is examined. Second, the concept of intentionality is looked over. Third, the concept of responsibility is sketched, as well as those of freedom, the idea of humanity, history, and the task of philosophy.
\end{abstract}

Keywords:

Husserl, phenomenology, responsibility, evidence, Crisis 
Ich mußte philosophieren, sonst konnte ich in dieser Welt nicht leben.

Husserl, citado por Mappe Heidegger en Schuhmann (1981, 344).

El concepto de responsabilidad plasmado por Husserl en la Crisis está estrechamente vinculado a su idea de racionalidad, pero tiene, además, un sentido particular respecto al filósofo. A grandes rasgos, podemos decir que, desde su perspectiva, la responsabilidad consiste en una toma de conciencia del papel de la subjetividad qua trascendental, lo que se traduce en una orientación hacia la evidencia experimentada (Husserl 2002, 28, 34 [Hua XXVII, 26, 32]). Precisamente, la tarea de la filosofía es descrita como la búsqueda de la fundamentación última de todo sentido y validez, la cual también implica un retorno a la evidencia. En la obra de Husserl podemos encontrar tres sentidos de este concepto, los cuales corresponden a los tres momentos de la delimitación de lo inmanente y trascendente. La Crisis describe el tercero, el cual concibe dentro del ámbito inmanente no solo lo dado actual, sino lo dado posible; $y$, por lo tanto, este también sería fuente de evidencia (Husserl 1991, § 46, 167; § 48, 174-175 [Hua Vl, 161-162, 169]; San Martín 2008, 63 ss.).

La mencionada tarea corresponde a la idea de la filosofía como profesión (Beruf) o, mejor, vocación (Berufung), esto es, la realización del conocimiento universal, cuyo valor es sentido por el filósofo, generando una tendencia hacia él, de forma que tal realización es un llamado (Ruf) para el filósofo: una suerte de demanda a la que tiene que responder (Husserl 1991, § 7, 17 [Hua Vl, 15]). En su búsqueda de la fundamentación de tal conocimiento y mediante el método fenomenológico (epojé y reducción trascendental), el filósofo ha de meditar (besinnen), a partir de su experiencia en primera persona, sobre el papel trascendental de la subjetividad, la cual descubre como una intersubjetividad trascendental. En el presente trabajo defenderé cómo la discusión de Husserl relativa a la tarea e idea de la filosofía conlleva la descripción de la fenomenología como una automeditación (Selbstbesinnung) de la humanidad y, respectivamente, del filósofo como un "funcionario de la humanidad" (Husserl 1991, § 7, 18; § 73, 280 ss. [Hua VI, 15, 272 ss.]), en tanto siente su valor, y adquiere la "responsabilidad última" por la autenticidad de la misma (Husserl 1991, § 35, 144 [Hua VI, 140]). 
Empezaremos con las diferentes versiones de la delimitación de lo inmanente y trascendente, y los correspondientes sentidos de evidencia. En una segunda parte describiremos la concepción de la intencionalidad de Husserl como una dinámica teleológico-normativa. Finalmente, en la tercera parte, exploraremos el tema de la responsabilidad, y algunos elementos relacionados a ella: la libertad, la humanidad, la historia, y la filosofía como idea y su correspondiente tarea.

\section{§1. La evidencia: entre lo dado y lo posible}

A lo largo de la obra de Husserl, podemos encontrar tres sentidos de lo inmanente y trascendente que corresponden a tres diferentes consideraciones sobre la evidencia. En la Crisis encontramos el tercero, así como la crítica a los anteriores. Antes de caracterizarlo, tenemos que entender cuál es la relación entre el par inmanente/trascendente y la evidencia. La distinción entre lo inmanente y trascendente designa dos ámbitos: el de la experiencia o vivencia y aquel externo a ella, su objeto (en sentido amplio) (San Martín 2008, 63). La evidencia, entendida como experiencia de la verdad, es aquella en la cual lo mentado en la intención es intuido y se circunscribe al ámbito inmanente'. En primera instancia, esta descripción nos puede remitir a un psicologismo, en tanto la verdad parece ser reducida a la experiencia subjetiva. Mas, justamente, el mérito de la consideración de Husserl es dar una salida al problema del enigma de la relación entre inmanencia y trascendencia describiendo su correlación, en la que se enfatiza el papel constituyente del sujeto como dirigido a los objetos y se reconoce,

1 Ya en el primer volumen de las Investigaciones lógicas, Husserl señala que "[l]a evidencia no es otra cosa que la 'vivencia' de la verdad. [...] la verdad es una idea cuyo caso individual es vivencia actual en el juicio evidente. Pero el juicio evidente es la conciencia de algo dado originariamente" (Husserl 2006, §51, 162 [Hua XVIII, 193]). En el epílogo de Ideas I, Husserl describe la evidencia y su vínculo con la inmanencia señalando que: "manteniendo en forma consecuentemente exclusiva la dirección de la experiencia a lo experimentable de modo puramente interno, a lo que me es fenomenológicamente 'accesible', tengo una esencia propia conclusa en sí, en sí misma conexa. A ella pertenece toda experiencia real y posible por medio de la cual está ahí para mí el mundo objetivo, con todas las verificaciones de experiencia en que se verifica para mí, aunque su validez nunca haya sido científicamente examinada" (Husserl 2013, 476 [Hua V, 149]). Como veremos, estas dos nociones de la evidencia corresponden al primer y tercer sentido de la inmanencia; sin embargo, la caracterización general de la evidencia como experiencia de la verdad se mantiene. 
a la vez, el papel de los cambios en el polo objetivo en las constituciones de sentido del sujeto; de esta forma, se cierra el abismo establecido por la modernidad entre uno y otro ámbito (Husserl 1991, §46, 167-169 [Hua $\mathrm{Vl}, 161-163])$. Como veremos, el tercer sentido de estos conceptos (inmanencia, trascendencia y evidencia) logra la mencionada salida al enigma describiendo la trascendencia de la inmanencia.

Los dos primeros sentidos del par inmanencia/trascendencia delimitan un ámbito de la inmanencia reducido frente a lo que será el tercer sentido defendido en obras como la Crisis. El primer sentido - presente en las obras Filosofía de la aritmética (1891) e Investigaciones lógicas (1900-1901) — solo incluye en el ámbito inmanente la experiencia y sus ingredientes (nóesis), de manera que lo trascendente es el objeto intencional (nóema): su dación actual, sus posibles daciones y lo no dable (Husserl 1929, §11, 495 [Hua XIX/1, B370-B378]; San Martín 2008, 64)². Respectivamente, la evidencia es entendida según el ideal cartesiano de adecuación entre el pensamiento y lo pensado. En la Crisis, Husserl describe este primer sentido en su crítica al modelo cartesiano y sus consecuencias en las propuestas de Locke (1991, § 19, 85; § 22, 88 ss. [Hua VI, 83, 86 ss.]).

El segundo sentido - presente en las obras La idea de la fenomenología (1907) y Lecciones de lógica y teoría del conocimiento (1906-1907)— amplía el ámbito de lo inmanente. Así, incluye, además de la vivencia, el objeto actualmente dado en ella, mas sigue considerando lo posiblemente dado —esto es, el horizonte de daciones posibles - y lo imposible de ser dado como trascendentes (San Martín 2008, 64).

La crítica a estos primeros sentidos se basa en que ninguno describe correctamente la relación entre los ámbitos inmanente y trascendente, lo cual implica que no dan cuenta del conocimiento. En efecto, si, como en el primer sentido, marcamos el límite entre la mera experiencia y el objeto al cual se dirige la intención, no tenemos cómo garantizar su vínculo, nos quedamos

2 En el segundo volumen de las Investigaciones, Husserl señala que "la expresión de objeto inmanente [...] designa la peculiaridad esencial de las vivencias intencionales; y asimismo por la expresión escolástica sinónima: in-existencia intencional o mental de un objeto" (1929, inv. V, § 11, 493 [Hua XIX/1, B372]). 
en la duda escéptica de si acaso nuestros contenidos mentales representan adecuadamente lo externo y no son meras apariencias subjetivas (Husserl $1991, \S 63,230$ [Hua Vl, 223]). En efecto, parece que hay un divorcio entre el contenido de la vivencia, calificado de inexistente, meramente mental, y el objeto dado en esta, que ha de ser existente y objetivo.

Asimismo, desde el segundo sentido, al incluir tanto lo posible como imposiblemente dado en el ámbito trascendente, se obvia una importante diferencia. Si incluimos lo dado del objeto intencional en el ámbito inmanente, logramos establecer un vínculo, pero limitado a lo actual, de forma que las posibilidades de dación no podrían estar incluidas en el conocimiento, lo cual no se condice con nuestra experiencia.

La tercera delimitación entre lo inmanente y trascendente - presente en obras como Problemas fundamentales de la fenomenología (1910-1911), Ideas I (1913), las Meditaciones cartesianas (1929) y la Crisis (1936) — incluye en el primero la experiencia vivida y tanto lo dado realmente en aquella experiencia como lo posiblemente dado (San Martín 2008, 64). Esto es, del lado del objeto, incluye aquello que mentamos a partir de la dación, una posibilidad real (Bernet 2004, 123-125). Esta tercera delimitación tiene dos periodos. La descripción brindada por Husserl pasa por una primera etapa estática - correspondiente a Problemas fundamentales de la fenomenología (1910-1911) e Ideas I (1913) - y luego una genética — propiamente plasmada en la Crisis (1936), pero ya presente en Meditaciones cartesianas (1929) — , en la que queda mucho más claro el carácter abierto de la intencionalidad, ya sugerido en Ideas I con la mención de las ideas en sentido kantiano (Husserl 2013, §83, 275-276 (Hua III/1, 166-167), incluyendo los temas del tiempo y la historia.

En la Crisis, basándose en el caso de la percepción, Husserl sostiene que en ella "[...] ya tengo la plena consciencia de la cosa, al igual que ya en la primera ojeada la veo como esta cosa. Viendo, la imagino [meinen] constantemente junto con todos los lados que en modo alguno me están dados, tampoco bajo la forma de un hacer presente previo intuitivo. Así pues, la percepción tiene 'consciencialmente' en cada caso un horizonte perteneciente a su objeto" (Husserl 1991, § 45, 166 [Hua VI, 161]). Las daciones 
posibles forman parte del horizonte interno del objeto (Husserl 1991, § 47, 171 [Hua Vl, 165]). Frente a este, el horizonte externo es el fondo sobre el que se destacan los objetos, siendo el mundo el horizonte final u horizonte de horizontes (Husserl 1991, § 38, 153 [Hua Vl, 148]).

Al discutir el ámbito inmanente explorado por el psicólogo, Husserl mantiene que "[...] aquí encuentra por doquier no sólo intenciones, sino los 'objetos intencionales', contenidos correlativamente en ellas [...]. No son partes reales de la intención, sino algo mentada en ella, su sentido respectivo, y esto en modalidades que sólo tienen sentido para cosas tales como 'sentido'"' (1991, § 70, 253 [Hua Vl, 245]). La última oración enfatiza la mencionada trascendencia de la inmanencia, ya que el objeto intencional (sus daciones actuales y posibles) es incluido dentro del ámbito inmanente, pero, como advierte Husserl, de una manera particular: si bien conocemos la cosa misma, y no una mera representación mental de ella, aquella se puede mentar y darse de diversas maneras, en diferentes aspectos. Según el fenomenólogo, lo trascendente es aquello no dado ni dable, el objeto (real o ideal) absolutamente completo: "'La' cosa misma es en verdad aqueIlo que nadie ha visto en tanto que real, puesto que, más bien, está constantemente en movimiento y es constantemente — para cualquiera - la unidad consciencial de la abierta e infinita multiplicidad de las cambiantes experiencias y cosas de experiencia propias y ajenas" (Husserl 1991, § 47, 173 [Hua Vl, 167]). En contraste con la división kantiana entre fenómeno (experiencia) y noúmeno (lo en sí, inalcanzable, al otro lado del abismo), para Husserl lo trascendente está más allá de la dación, funciona como una idea reguladora, pero no hay tal abismo y, por lo tanto, el conocimiento es posible (1991, § 45, 166 [Hua VI, 160]).

En esa línea, la evidencia no es pensada según el ideal cartesiano de adecuación perfecta, ya que incluye lo posible. Así, la adecuación es reemplazada por la apodicticidad, la cual puede incluir inadecuaciones actuales, y si bien implica una certeza en el más alto grado, es corregible (1979, § 6, 52 [Hua I, 56]). En la Crisis, al discutir las Investigaciones, la evidencia es descrita 
como una "autodación original universal" en la que tenemos experiencia de la verdad (Husserl 1991, § 68, 245 [Hua VI, 237]) 3.

En esta concepción Husserl resalta la condición temporal de la constitución de cualquier objeto, así como de la propia vida. Como veremos en la siguiente parte, tal condición temporal tendrá consecuencias para la descripción de la intencionalidad y, por lo tanto, de la conciencia - específicamente, será una de sus estructuras-:

Ninguno de estos actos, ni ninguna de las valideces comprendidas en el Yo, está aislado [...] lo en cada caso activamente consciente y, correlativamente, los activos contenidos de consciencia, estar-orientada-a, estar-ocupado-con, están bañados por una atmósfera de valideces mudas, ocultas, pero co-funcionantes, están bañados por un horizonte viviente en el que el Yo actual también puede adentrarse cuando le plazca, reactivando antiguas adquisiciones, asiendo conscientemente sorprendentes ideas aperceptivas, moviéndose y caminando en intuiciones (Husserl 1991, § 40, 157 [Hua Vl, 152]).

\section{§2. La intencionalidad en la Crisis}

çUna vez aclaradas estas diferentes delimitaciones de los ámbitos inmanente y trascendente, así como su relación con la idea de evidencia, podemos discutir la noción de la intencionalidad. Según Husserl, esta es la característica principal de la conciencia: su estar-dirigida-a, y describe su estructura como una teleológico-normativa (1991, §50, 180-181 [Hua VI, 174]). Entonces, la intencionalidad se orienta — no solo en el sentido de dirección sino de tendencia (aspecto volitivo) ${ }^{4}$ - , hacia un fin, un ideal de plenitud que, a su vez, funciona como norma. Aquí ya resalta un elemento importante de

3 “Toda acreditación imaginable reconduce a estos modi de la evidencia, puesto que lo 'ello mismo' (del correspondiente modus) reside en estas mismas intuiciones como lo realmente experimentable y acreditable intersubjetivamente, y no es ninguna substrucción mental, mientras que, por otra parte, una substrucción tal, en la medida en que pretende en general la verdad, sólo puede poseer una verdad real precisamente por medio de la retrorreferencia sobre tales evidencias" (Husserl 1991, § 34d, 134 [Hua VI, 130]).

4 Cabe aclarar que, si bien esta tendencia pertenece a los actos volitivos, no es voluntaria, es decir, no es propiamente consciente o activa, sino que ya está presente en el estrato pasivo-instintivo, el cual tiene un carácter dinámico (Hua XIV, 56, 69, 333; Hua XV, 201; Hua XXXVII, 146; Hua XLII, 102, 118, 225). 
la propuesta de Husserl, que funciona como un presupuesto de sus ideas: la unidad de la razón. Según Husserl, si bien es posible distinguir diferentes tipos de actos (cognitivos, emocionales y volitivos) en una descripción de la experiencia, esto no significa que esta se dé efectivamente de esta manera fragmentaria, ya que la razón es unitaria.

Si bien el retrato de Husserl de la intencionalidad empieza en el ámbito cognitivo, esta termina describiendo toda la vida subjetiva ${ }^{5}$. Desde sus análisis estáticos, el orden de fundación de los actos que propone Husserl describe que los actos emocionales (sentir el valor o valorar) fundan los actos volitivos, y tales actos emocionales, a su vez, se fundan en los actos de presentación y pensamiento. No obstante, esta unidad no se limita a la articulación de los diferentes actos, sino también al enraizamiento de lo activo en lo pasivo. La génesis pasiva describe que la intención como tendencia se proyecta desde el dominio pasivo, donde lo afectivo tiene un papel prominente, hacia el activo: los elementos que componen los diversos actos descritos por Husserl están presentes en el primero, solo que indiferenciados (Husserl 2005, § 4, 39-40 [Hua IV, 10]; Hua XI, 418; Hua XV, 385; Hua XXVIII, §§ 3-4, 12d, 17, 178, 343; Hua XXXVII, 181; Walton 2017, 4). De esta manera, podemos decir que el valorar se fundamenta en la vida afectiva, y que esta, en gran medida, impulsa y motiva la vida activa del sujeto ${ }^{6}$.

La dinámica de la intencionalidad puede ser descrita en tres momentos o procesos: la mención, la intuición y la síntesis de plenificación. Si bien estos tres son comunes a todos los actos intencionales (cognitivos, emocionales y volitivos), corresponden a la descripción del análisis estático, el cual parte del objeto intencional como constituido en actos intencionales

5 "das transzendentale Bewußtsein ist ja von vornherein universales, alles Bewußtsein, auch die gesamte Gemüts- und Willens- intentionalität mitumspannend; alles das nicht im Nebeneinander, sondern in seiner — wie sich zeigt — untrennbaren Einheit" (Hua VIII, 504).

6 "Este objeto de valor, que en su sentido objetivo encierra el carácter de la valiosidad como propio de su ser-así, es el correlato de la captación teórica del valor. es por tanto un objeto de nivel superior. Observamos que el juicio de valor original-general, hablando en términos generales, toda conciencia que originariamente constituye un objeto de valor como tal, posee en sí necesariamente un componente que pertenece a la esfera emotiva" (Husserl 2005, §4, 39 [Hua IV, 9]). Sobre el tema de los actos emocionales y el valor en la fenomenología de Husserl, véase Quepons 2016; Chu 2013; Walton y otros 2017. 
y experiencias de evidencia correspondientes (1991, § 48, 174-176 [Hua VI, 168-170]), y son, por lo tanto, abstractos, ya que, como mencioné, en nuestra experiencia en primera persona no se dan diferenciados.

En la Crisis, Husserl describe la estructura de la intencionalidad tomando el ejemplo de los actos perceptivos, que forman parte del primer tipo de actos, los cognitivos, mas esta explicación es análoga a la de cada tipo de acto. Lo importante de la estructura transversal a los tipos de actos es la dinámica descrita entre la multiplicidad y unidad: "Todo ente indica una universalidad ideal de formas de dación experimentantes reales y posibles, cada una de las cuales es aparición de este único ente y, ciertamente, de modo tal que toda experiencia concreta real realiza, a partir de esta multiplicidad total, un transcurso de formas de dación consonantes y que satisface continuamente la intención experimentadora" (1991, §48, 175; § 43, 165 [Hua Vl, 169, 160]).

Por ejemplo, si bien percibimos objetos como unidades inalteradas, fijas, tal acto se da, a su vez, a través de un proceso temporal de múltiples percepciones particulares (Husserl 1991, §45, 165 [Hua Vl, 160]). Esta multiplicidad tiene dos niveles: (1) la multiplicidad de tipos de actos que nos presentan diferentes aspectos del objeto intencional (en el caso de los actos cognitivos, percepciones visuales, táctiles, olfativas; intuiciones categoriales $\left.{ }^{7}\right), y(2)$ la multiplicidad de grados de plenificación implicados en cada acto, ambos respecto al mismo objeto unitario (1991, § 45, 165-166 [Hua Vl, 160-161]). Cada aspecto particular "se me presenta en una síntesis continua" y es una "forma de representación \{Darstellungsweise\}" del objeto unitario (1991, § 45, 166 [Hua Vl, 161]). Esta multiplicidad nos remite al retrato de la distinción de lo inmanente y trascendente, así como aquel de la intencionalidad y su carácter tendencial. Como mencionamos, Husserl supera el enigma del conocimiento entre lo subjetivo y objetivo al señalar que podemos conocer las objetividades, aunque la objetividad plena es trascendente a

7 Análogamente, esta multiplicidad también caracteriza a los emocionales -el sentir el valor - y volitivos - tender hacia una meta, desear, tomar una decisión, etc.

8 Uso llaves para mis agregados, para diferenciarlos de los corchetes y comillas españolas que se usan en la obra. 
la intencionalidad, ya que tales se nos dan de múltiples maneras, en sus diferentes actos.

\section{§2.1. Mención vacía e intuición}

La mención vacía es la referencia a algo (en sentido amplio, un objeto o un aspecto del mismo) que no está actualmente dado. Frente a esta, la intuición es la captación de lo actualmente dado. En la Crisis, Husserl sostiene respecto a la percepción que “[...] lo auténticamente percibido \{intuido\}'remite' a una multiplicidad sistemática de posibles representaciones conforme a la percepción que le pertenecen consonantemente" (1991, § 47, 171 [Hua VI, 165]). Como podemos apreciar, la intención e intuición son diferentes tipos de actos, mas generalmente se dan juntos ${ }^{9}$. En cada percepción tenemos una intención intuitiva del aspecto particular que efectivamente percibimos junto a una mención vacía de sus otros aspectos y el objeto unitario, absoluto, del cual nunca tenemos una intuición completa: “[...] mientras que la superficie está dada actualmente imagino [meine] más de lo que ella ofrece" (Husserl 1991, § 45, 166 [Hua VI, 160]).

Así, a pesar de que no tengamos tal intuición completa, ella está presente en cada una de nuestras percepciones parciales, como una "certeza-de-ser de esta cosa para la que todos los lados son propios al mismo tiempo" (Husserl 1991, § 45, 166 [Hua Vl, 160]), y tiene una función normativa, indicándonos el óptimo de su dación, en forma de una anticipación. La mención vacía e intuición se vinculan en tanto esta última plenifica la primera, y es en esa plenificación del sentido mentado que experimentamos la evidencia. Al caracterizar al "yo" como tema de investigación, Husserl describe tal tendencia a la plenificación como esencial a la intencionalidad:

9 En las Investigaciones, al discutir el acto intencional y su contenido, Husserl señala que "[s]egún que este contenido funcione como representante signitivo puro, o intuitivo puro, o como ambas cosas a la vez, será el acto signitivo puro o intuitivo puro mixto" (Husserl 1929, inv. VI. § 25, 662 [Hua XIX/2, 88]). Husserl usa diversos términos para referir a la mención vacía, siendo la intención signitiva uno de estos (Rizo-Patrón 2015, 246, 254, 273). 
[...] el idéntico realizador, a su modo, de todas las valideces, como el Yo que tiende-a; como el Yo orientado en el cambio de las formas multiescalonadas de aparición "a través de ellas" hacia el polo unitario, esto es, orientado hacia la meta ambicionada desde él (su proyecto), meta mentada previamente con mayor o menor precisión y claridad y que se satisface sólo siendo-deviniendo de fase en fase - satisfaciendo su intención- (1991, § 50, 180 [Hua Vl, 174]).

En esta descripción, como adelantamos, resalta el elemento temporal, en tanto la multiplicidad de daciones $y$, correlativamente, los actos se extienden a lo largo de nuestra experiencia personal ${ }^{10}$. Notamos que en toda percepción particular, "[...] en tanto que la de un objeto extenso y duradero, hay una continuación de lo aún-consciente, fluyente, de ninguna manera intuitivo por más tiempo, una continuidad de 'retenciones' y, en otra dirección, una continuidad de 'protenciones"' (Husserl 1991, § 46, 168-169 [Hua Vl, 163]). Impresión originaria (Urimpression), retención y protención son momentos abstractos de la percepción, es decir, son relativos a un presente y se relacionan con la apercepción. Lo previamente dado a la intuición, esto es, lo efectivamente percibido en algún momento pasado, también está mentado en las subsiguientes intuiciones; es decir, no solo anticipamos el óptimo según lo dado actual, sino que conservamos lo dado previamente en la síntesis de las percepciones particulares:"[...] las múltiples adquisiciones de la vida activa anterior no son sedimentaciones muertas, sino que el trasfondo que permanece plenamente inadvertido (por ejemplo, del campo perceptivo), trasfondo siempre co-consciente, pero momentáneamente irrelevante, este trasfondo co-funciona según sus valideces implícitas" (Husserl 1991, § 40, 157 [Hua VI, 152]).

Es a través de este proceso que conocemos los objetos, conservando lo previamente dado, anticipando su óptimo, y corrigiendo el sentido según el curso de las intuiciones:"[...] en el cambio continuo del ver el lado visto cesa de ser visto realmente, pero es 'conservado' y 'juntado' con los lados anteriormente conservados, y así 'aprendo' a 'conocer' la cosa" (Husserl 1991, § 45, 166 [Hua VI, 160]).

10 "La percepción sólo se refiere al presente. Pero se presupone que este presente tiene tras sí un pasado infinito y ante sí un futuro abierto" (Husserl 1991, §46, 168 [Hua VI, 163]). 


\section{§2.2. Síntesis de plenificación}

Entonces, la plenificación del sentido, antes que un acto particular, es un proceso temporal que reúne diferentes actos. Como señalamos, en la percepción, tomando el ejemplo de la Crisis, nunca nos es dado el objeto totalmente, sino por múltiples escorzos y en diferentes grados de plenificación, los cuales, sin embargo, relacionamos al mismo como su representación, en una síntesis de identificación o unificación (Husserl 1991, § 45, 167 [Hua Vl, 161]). En esta se articulan las daciones actuales, correlativas a la intuición, y las posibles, correlativas a las menciones vacías, las cuales incluyen daciones previas y anticipaciones, tanto de otros escorzos como del objeto total. De forma que el óptimo anticipado en cada acto cumple una función normativa en la unión de la multiplicidad de actos y sus daciones correlativas ${ }^{11}$.

Cabe aclarar que la síntesis de plenificación no se da en un proceso siempre ascendente, sino que está abierto a decepciones y correcciones: "[...] a menudo se llega a la ruptura de esta consonancia: ser se transforma en [...] \{ilusión\}, o tan sólo en ser dudoso, en mero ser posiblemente, en ser probable, en-no-ser-sin-embargo-mera-apariencia-nula, etc. Entonces la apariencia se resuelve mediante 'corrección', mediante la modificación del sentido en el que se había percibido la cosa" (Husserl 1991, § 47, 170 [Hua $\mathrm{VI}, 164]$ ). Conforme intuimos diversas daciones, modificamos el sentido inicialmente mentado, ya sea plenificándolo o corrigiéndolo, ajustando, a su vez, nuestras anticipaciones.

Asimismo, en esta síntesis también el elemento intersubjetivo es esencial. Ya en los parágrafos 48-49 de la Crisis Husserl advierte sobre el rol de la mancomunación en la constitución, la cual termina siendo considerada intersubjetiva: "la subjetividad sólo en la intersubjetividad es lo que es: Yo

11 "Esto \{la síntesis\} no sucede como amalgamiento externo; sino que las representaciones, en tanto que portando en sí 'sentido' en cada fase y mentando algo, se ligan en la dirección de un progresivo enriquecimiento de sentido y de una continua formación de sentido, en la que aquello que ya no aparece continúa vigente como algo aún conservado, y en la que la mención previa anticipadora de un transcurso continuo, la expectativa previa de lo 'venidero', se satisface $y$, al mismo tiempo, se determina de una forma más próxima. De este modo, todo es recogido en la unidad de validez o bien en lo uno, la cosa" (Husserl 1991, § 45, 167 [Hua Vl, 161]). 
que actúa constitutivamente" (Husserl 1991, § 50, 181 [Hua VI, 175]). Así, las síntesis de plenificación a nivel subjetivo-individual están condicionadas por las síntesis intersubjetivas, las cuales no solo las hacen posibles, sino que las orientan (Husserl 1991, §50, 182 [Hua VI, 175]). Además, las síntesis intersubjetivas, en gran medida, son las que posibilitan la corrección: "En dirección hacia la diferencia entre cosas 'originalmente propias' y cosas que sabe 'por empatía' que otro experimenta en el cómo de las formas de aparición, e incluso en dirección hacia la posibilidad de disonancias entre las concepciones propias y las que sabe por empatía", es decir, en ellas, "aquello que cada uno experimenta realmente originaliter como cosa de percepción se transforma para cada cual en mera 'representación de', en 'aparición de', i.e.: de lo único que es objetivamente" (Husserl 1991, § 47, 173 [Hua VI, 167]). Por lo tanto, toda constitución implica que la objetividad lo es tal para toda subjetividad y no solo una individual.

Así, la estructura de la intencionalidad implica una dinámica temporal entre la multiplicidad de momentos parciales y la unidad a la que se tiende, a nivel subjetivo e intersubjetivo, cuya orientación hacia aquel fin que funciona como norma es aquello que articula tal multiplicidad en una unidad.

\section{§3. Responsabilidad en la Crisis}

Podemos resumir la cuestión de la responsabilidad con la pregunta de si estamos dispuestos a hacernos cargo de los sentidos que mentamos y bajo qué justificación (Dodd 2004, 9); y, para responder a ella, hemos de recurrir a la evidencia original, intuida en primera persona. Así, el concepto de responsabilidad refiere a la actitud de la búsqueda de fundamentación última del conocimiento, es decir, la posibilidad de hacer afirmaciones de sentido válidas, y surge como parte de nuestra automeditación (Selbstbesinnung), nuestro (re)conocernos como subjetividad trascendental, esto es, constituyente de sentido. Tal reconocimiento del carácter trascendental de la subjetividad implica la toma de conciencia de nuestra responsabilidad, no solo respecto a los sentidos que constituimos activamente, sino también respecto a aquellos previamente instituidos, que reactivamos cada vez que apelamos a ellos (Husserl 1991, § 52, 186-187 [Hua VI, 180-181]). 
Para discutir este tema y su vínculo con lo presentado sobre la evidencia y la dinámica de la intencionalidad, tenemos que discutir la concepción de la libertad de Husserl - tanto en general como en el caso específico del filósofo-, la cual va de la mano con su idea de humanidad. Asimismo, exploraremos el papel de la historia y su relación con la institución de la idea de la filosofía como una tarea, el papel de la Besinnung, y cómo se relaciona esta tarea con el llamado a la búsqueda de fundamentación última.

\section{$\S 3.1$. Libertad}

Husserl vincula la idea de la libertad a la razón: "El espíritu e incluso sólo el espíritu es siendo él mismo en sí mismo y para sí mismo, es autónomo, y en esta autonomía, y sólo en ella, puede ser tratado de manera verdaderamente racional, de manera verdadera y radicalmente científica" (1991, 355 [Hua $\mathrm{Vl}, 345])$. En Renovación, considera dos dimensiones de esta libertad que complejizan su retrato: (1) un ser-libre-de, en tanto el ser humano tiene la "peculiaridad esencial de 'actuar' libre y activamente desde sí mismo, desde su yo-centro, en lugar de estar entregado pasivamente a sus impulsos (tendencias, afectos)" (Husserl 2002, 24-25 [Hua XXVII, 24]); y (2) un ser-libre-para, "una actividad auténticamente 'personal"' en la que "el hombre tiene experiencia [...] piensa, valora, interviene en el mundo circundante" y se orienta hacia una vida en la evidencia (Husserl 2002, 25 [Hua XXVII, 24]; cfr. Hart 1992, 94). Entonces, ser libre, tomar nuestras propias decisiones, implica basarnos en la evidencia que experimentamos (Hart 1992, 96), lo cual advierte su carácter esencialmente crítico: “Libertad es [...] una expresión para la facultad y sobre todo para el hábito adquirido de tomar una postura crítica a propósito de lo que, primeramente sin reflexión, se da a la conciencia como verdadero, como valioso, como debiendo ser en sentido práctico" (Husserl 2002, 70 [Hua XXVII, 63]).

Además de este sentido de libertad de la voluntad, hay uno de la razón en general. Precisamente, en la Crisis, Husserl ofrece un detallado cuadro de la razón y el tipo de vida que supone, en el cual resaltan los elementos 
de la libertad, meditación (Besinnung) ${ }^{12}$, evidencia y responsabilidad ${ }^{13}$. Este retrato de la vida subjetiva, aclara en seguida, se aplica al individuo y a la comunidad, en tanto la vida personal está marcada por una tendencia hacia aquella "[...] necesidad [...] de hacer llegar la razón personal individual a una realización cada vez más perfecta tan sólo como razón comunitaria personal" (Husserl 1991, §73, 280 [Hua Vl, 273]). En efecto, la relación entre subjetividad e intersubjetividad, a nivel trascendental, y mónada personal y comunidad intermonádica, a nivel concreto, es considerada paradójica por Husserl, ya que, desde el orden del conocimiento, la subjetividad es primera, porque en nuestra experiencia todo fenómeno es relativo; mas, desde el orden del ser, la intersubjetividad en tanto trascendental —el polo constituyente de la correlación universal - y el mundo —el polo constituido- preceden a la subjetividad (Husserl 1991, § 72, 273-274 [Hua Vl, 265-266]). Es decir, el movimiento de la constitución entre lo subjetivo e intersubjetivo no es unilateral, sino recíproco.

Ahora, el filósofo vive esta libertad de una manera específica, al ser un elemento clave del método fenomenológico, específicamente, de la epojé trascendental, y, por lo tanto, del descubrimiento del a priori de la correlación. Husserl sostiene que "[...] con ella \{la epojé\} la mirada del filósofo se torna de hecho plenamente libre por vez primera y, sobre todo, libre de la ligazón interna más fuerte y más universal y, en esta medida, más oculta, a

12 Cabe resaltar un cambio en el vocabulario de Husserl respecto a este término. En el periodo estático de su obra - centrado en la descripción de la estructura, funciones y modos de la intencionalidad—usa el término alemán "Reflexion" (Husserl 2013, §45, 176-178; §§ 77-79, 250 ss.; § 108, 341 [Hua III/1, 83 ss., 144 ss., 221]). En cambio, en el periodo genético, usa el término "Besinnung", haciendo mayor énfasis en el aspecto ético-existencial que en el epistemológico (Husserl 1979, § 61, 221 [Hua I, 174]; Husserl 1991, § 7, 18; § 73, 280 ss. [Hua Vl, 15-16, 272 ss.]; Hua Vl, 489). Para diferenciar ambos sentidos, en este trabajo usamos "meditación" para traducir Besinnung y "automeditación" para traducir Selbstbesinnung. La traducción de la Crisis que estamos empleando ha sido correspondientemente modificada.

13 "La razón es lo específico del hombre en tanto que ser que vive en actividades y habitualidades personales. En tanto que vida personal, esta vida es un constante devenir en una constante intencionalidad del desarrollo. [...] La vida humana personal discurre en niveles de automeditación y autorresponsabilidad, [...] hasta la aprehensión consciencial de la idea de autonomía, de la idea de la capacidad volitiva de decisión para configurar con autorresponsabilidad universal toda su vida personal en la dirección de la unidad sintética de una vida; correlativamente, para configurarse a sí mismo en la dirección de un Yo verdadero, libre, autónomo, el cual <busca> realizar la razón que le es innata, el esfuerzo por ser fiel a sí mismo, por poder permanecer idéntico consigo mismo en tanto que Yo-razón" (Husserl 1991, § 73, 280 [Hua VI, 272]; cfr. Hua Vl, 486). 
saber: libre de aquella ligazón de la dación previa del mundo" (1991, § 41, 159 [Hua Vl, 154]). El vínculo entre el método fenomenológico y la libertad se justifica en una comprensión de la génesis de la filosofía como un cambio de actitud, y del aspecto práctico-volitivo de la epojé "[...] como una actitud habitual por la que nos decidimos de una vez por todas" (Husserl 1991, § 40, 159 [Hua Vl, 154, 239]). Aquí se hace referencia al retrato de la razón como una unidad, el cual, como dijimos, funciona como presupuesto de las ideas de Husserl presentadas en este trabajo. En efecto, la libertad teórica del método fenomenológico, de la filosofía, no se desvincula del aspecto volitivo-práctico, ya que su realización depende de actos volitivos: toda dimensión de la subjetividad está atravesada por tal aspecto (Husserl 1980, § 78, 340; Husserl 2002, 42 [Hua XXVII, 40]; Hua XXIV, 218).

Como sostiene Steinbock, sin embargo, hay que entender la libertad implicada en la epojé como un movimiento libre del pensamiento que, como tal, no corresponde a ninguna motivación proveniente del mundo de la vida, ningún interés pragmático: estas pueden ser asociadas, a posteriori, en el análisis desarrollado a partir de la automeditación (Steinbock 2019, $4,7)$. En esa línea, la motivación de la meditación llevada a cabo en la obra es, efectivamente, la crisis que, según Husserl, Europa atravesaba, mas tal meditación es posible por el libre movimiento de nuestra conciencia: la crisis es solo una ocasión.

Ahora, a este tipo de libertad le corresponde una responsabilidad particular: "La responsabilidad enteramente personal por nuestro propio y verdadero ser como filósofos en nuestra vocación personal más íntima entraña y lleva también en sí la responsabilidad por el ser verdadero de la humanidad, que no es sino en cuanto ser tendente a un telos y que si puede ser realizado, lo puede únicamente por la filosofía, por nosotros, si es que somos seria y rigurosamente filósofos" (Husserl 1991, § 7, 18 [Hua Vl, 15]). La filosofía como vocación consiste en una responsabilidad máxima para con la humanidad.

Aquí hay varios elementos relacionados $y$, para aclararlos, tenemos que discutir el concepto de Besinnung o meditación, central para esta obra de Husserl, en tanto describe el ejercicio doble del fenomenólogo: como descripción que contempla distinciones esenciales $-\mathrm{y}$ no una mera 
meditación personal o apertura inicial - y como crítica, la cual implica un análisis que descubre la dación como una determinación de sentido y, por lo tanto, fuente de justificación (Dodd 2004, 3). Como menciona Steinbock, el método fenomenológico, entendido como un alejamiento de la ingenua actitud natural, es inherentemente crítico: "una liberación de los fenómenos tal que lo que es, en su profundidad propia, es lo que aparece tal cual es dado" $(2019,3)$. Esta crítica es guiada teleológicamente por la tarea correspondiente a la idea de la filosofía, que tiene un aspecto objetivo y subjetivo, esto es, el objetivo o propósito designado por la tarea (la actividad), y el estado del filósofo que se siente llamado, obligado (compelled) a realizarla (Dodd 2004, 64). Esta tarea y su correspondiente llamado se basan últimamente en el sentir del valor del conocimiento por parte del filósofo. Como mencionamos, según Husserl, lo práctico - esto es, las acciones y los actos relacionadas a ellas, como la decisión, tendencia, deseo, etc. - se funda en lo emocional —el sentir de los valores-, y esto, a su vez, en la actividad cognitiva (Husserl 1929, § 41, 578 [Hua XIX/1, B493-B494]; Husserl 2013, §37, 157; §116, 362; §117, 365 [Hua III/1, 66, 229, 242]; Husserl 2005, §4, 39 [Hua IV, 9]), pero últimamente toda la actividad se proyecta desde el estrato pasivo-afectivo. Entonces, que la tarea del filósofo sea la fundamentación del conocimiento implica que siente su valor y este motiva sus actos.

Husserl entiende la Besinnung como una toma de conciencia crítica, cuyo objeto es la plenitud o autenticidad del sentido del conocimiento y cuya justificación se descubre en la evidencia, la cual le da su carácter racional (Dodd 2004, 4-6). Ahora, esta toma de conciencia ya implica un compromiso con la afirmación de tal sentido y sus posibles reactivaciones: de ahí su vínculo con la responsabilidad (Dodd 2004, 8). Mas Husserl describe la Besinnung propia del filósofo como una Selbstbesinnung o automeditación (mismo). El uso del prefijo sí (mismo)- (Selbst-) enfatiza que esta meditación ( $y$, consecuentemente, el compromiso, la responsabilidad) no apela al sentido como independiente, sino a la subjetividad que lo constituye y sus posibilidades (Dodd 2004, 9). Husserl indica que, entonces, la Selbstbesinnung es "die Rückfrage nach dem Sinn, dem teleologischen Wesen des Ich"14, y no meramente un recuento de nuestra vida concreta (Hua VI, 511, n.).

14 Traducción sugerida: "el repreguntar sobre el sentido, sobre la esencia teleológica del Yo". 
En efecto, la Selbstbesinnung está íntimamente ligada a la fenomenología como método develador de la subjetividad qua trascendental: "[...] el éxito real de una filosofía transcendental depende a más no poder de la claridad automeditativa \{toma de conciencia de sí mismo, selbstbesinnlichen Klarheit\}" (Husserl 1991, § 42, 162 [Hua Vl, 156]) ${ }^{15}$.

La importancia de esta Selbstbesinnung nos remite a la idea de la filosofía como fundamentación última de todo conocimiento. El descubrimiento de la fenomenología trascendental es que la primera evidencia es la apodicticidad del ego en tanto subjetividad trascendental, la cual no es sino un aspecto del yo empírico o concreto que se mantiene oculto para la actitud natural (Husserl 1991, § 58, 215 [Hua Vl, 209]; cfr. Rizo-Patrón 2005, 290). Husserl detalla esto en el epílogo de Ideas I:

[...] me percato ahora también de que mi esencia propia fenomenológicamente conclusa en sí, es susceptible de ser puesta absolutamente, como el yo que soy, el cual confiere validez de ser al ser del mundo del que en cada caso hablo. El mundo es para mí y es lo que es para mí solo en tanto que adquiere sentido y validez verificada a partir de mi propia vida pura y de la vida de los demás que se descubre en la mía. Yo, en cuanto esta esencia absolutamente puesta [...] en adelante tengo dado exclusivamente [...] mi yo puramente como el yo que es en sí y que en sí experimenta el mundo, lo verifica, etc. (Husserl 2013, 476-477 [Hua V, 149]; cfr. 474 [Hua V, 147]).

Como mencionamos, la responsabilidad implicada en esta automeditación consiste, una vez reconocemos este carácter trascendental de la subjetividad, en hacernos cargo de nuestras constituciones y reactivaciones de sentido (Hua Vl, 489). Asimismo, ya que la subjetividad trascendental es, últimamente, una intersubjetividad trascendental (Husserl 1991, § 50, 181 [Hua Vl, 174]), esta responsabilidad también se extiende a los colectivos de diversos niveles de los cuales formamos parte: "Die Besinnung soll offenbar den Charakter der radikalen Verantwortung haben, und zwar als einer in seiner

15 En las Meditaciones cartesianas, Husserl escribe: "La meditación sobre sí mismo radical y la plenamente universal son empero inseparables entre sí $y$, al mismo tiempo, son inseparables del auténtico método fenomenológico de la automeditación \{Selbstbesinnung\} bajo la forma de la reducción trascendental [...]. La autoexplicitación universal y eidética significan, sin embargo, dominio sobre todas las posibilidades constitutivas concebibles 'innatas' al ego y a una intersubjetividad trascendental" (1979, § 64, 230 [Hua I, 179-180]). 
Person sich abspielenden, die doch den Charakter einer gemeinschaftlichen Verantwortung der gesamten philosophischen Gemeinschaft der Gegenwart annimmt" (Hua Vl, 489) ${ }^{16}$.

Vinculado al carácter infinito de la idea de la filosofía, la automeditación no es un momento específico, sino un tipo de actitud permanente propia del filósofo, que podríamos resumir como una problematización constante del sentido, la cual demanda ser renovada (Dodd 2004, 19). Tal demanda de renovación, sumada al componente intersubjetivo, implica un descubrimiento de la historicidad de la filosofía, esto es, entenderla como una realización histórica (Hua VI, 507), lo cual discutiremos más adelante.

\section{§3.2. La idea de la humanidad}

Como señalamos, la responsabilidad implicada en la automeditación del filósofo es relativa al problema de la autenticidad de la humanidad. Husserl señala que "Selbstbesinnung bleibt die ständige Funktion des zum vollen Menschen Werdens und das volle Menschentum Verwirklichens" (Hua $\mathrm{Vl}, 486)^{17}$. Esto es, la automeditación es esencial para la constitución de la unidad personal, a nivel individual y colectivo.

Precisamente por ello \{el descubrimiento de la subjetividad trascendental\} también da un nuevo sentido a la existencia humana, a su existencia en el mundo espacio-temporalmente dado con anterioridad, a su existencia en tanto que autoobjetivación de la subjetividad transcendental y de su ser, de su vida constituyente. Y como consecuencia ulterior da por resultado la autocomprensión última del hombre en tanto que responsable de su propio ser humano, de su autocomprensión en tanto que ser que está llamado a una vida en la apodicticidad. Y lo hace no sólo cultivando abstractamente y en sentido habitual una ciencia apodíctica, sino cultivando una ciencia que realice su ser concreto global en libertad apodíctica hacia una razón apodíctica, hacia una razón en toda vida activa suya —en la que es humanidad (Husserl 1991, § 73, 283 [Hua Vl, 275]).

16 Traducción sugerida: “La meditación debe tener manifiestamente el carácter de la responsabilidad radical, precisamente como una que tiene lugar en su persona, que empero asume el carácter de una responsabilidad comunitaria de toda la comunidad filosófica contemporánea". 17 Traducción sugerida: "La automeditación sigue siendo la función permanente del pleno devenir ser humano y de la plena realización de la humanidad". 
En efecto, el descubrimiento de la subjetividad trascendental qua intersubjetividad trascendental tiene efectos para la humanidad, en tanto su contraparte concreta. Husserl vincula su idea de la humanidad a la razón: "[...] si el hombre es un ser racional (animal rationale) lo es sólo en la medida en que su humanidad entera es una humanidad racional, una humanidad orientada de modo latente hacia la razón y de modo manifiesto hacia la entelequia accedida a consciencia de sí [...]" (1991, § 6, 16 [Hua Vl, 13]). Esta idea se remite a la consideración de Husserl sobre el origen de la filosofía y la figura espiritual de Europa en la antigua Grecia, concebida como un cambio de actitud, teleológicamente dirigido “[...] en la libre conformación de su existencia, de su vida histórica a partir de las ideas de la razón, en orden a tareas infinitas" (Husserl 1991, 328, 330, 335-336; § 33, 127 [Hua $\mathrm{VI}, 319,321,325-326 ; 124])$. Husserl sostiene que, además del cambio de actitud de la natural (espectador interesado en objetos del mundo) a la teorética (espectador desinteresado, que lleva a cabo la epojé respecto a lo práctico-mundano) (Husserl 1991, 341-342 [Hua VI, 331-332]), puede darse una síntesis entre ambas, "[...] de tal modo que la teoría (la ciencia universal) que se desarrolla en unitariedad cerrada y en epojé respecto de toda praxis se siente vocada \{berufen\} [...] a servir de un modo nuevo a la humanidad" (Husserl 1991, 338-339 [Hua VI, 329]). El resultado es

[...] un nuevo modo de formación de la comunidad y una nueva forma de comunidad duradera, cuya vida espiritual, que debe su condición de vida común al amor a las ideas, a la producción de las ideas y a la conducción ideal de la vida según normas, lleva en sí el horizonte de futuro de la infinitud. [...] infinitud de las generaciones que se renuevan desde y a partir del espíritu de las ideas (Husserl 1991, 332 [Hua VI, 322]).

Aquí resalta el aspecto crítico de la automeditación, ya que el develamiento de lo dado como producto de la subjetividad trascendental nos abre al cuestionamiento de los sentidos previamente sedimentados, en tanto ya no son vistos como necesarios, sino una posibilidad entre otras, producto de nuestras efectuaciones (individuales y colectivas), de las que somos responsables. Tal reconocimiento de la responsabilidad, según Husserl, implica (1) "una crítica de la humanidad misma y de los valores que la dirigen de modo tácito o expreso" $y$, consecuentemente, (2) "una praxis que apunta a elevar mediante la razón científica universal a la humanidad según normas 
veritativas de todas las formas, a transformarla en una humanidad nueva desde la raíz, capacitada para asumir una autorresponsabilidad absoluta sobre la base de conocimientos teóricos absolutos" (Husserl 1991, 339 [Hua VI, 329]).

Como vemos, entonces, tal idea de la humanidad es producto de la génesis histórica de la filosofía (Husserl 1991, 347-348 [Hua Vl, 337-338]). Pero, además, implica un cambio en nuestra comprensión de la historia pasada y proyección al futuro: "[...] una revolución de la historicidad, que es ahora la historia de la pérdida de devenir de la humanidad finita en el devenir hacia la humanidad de tareas infinitas" (Husserl 1991, 334 [Hua VI, 325]).

\section{§3.3. El papel de la historia}

Para Husserl, la filosofía no se hace desde el vacío: ser filósofo implica reconocerse - como producto de la Selbstbesinnung - en una comunidad actual y potencial de filósofos, cuya unidad reposa en su orientación hacia la realización de la idea de la filosofía. En efecto, la automeditación histórica de la filosofía llevada a cabo en la Crisis nos sirve de introducción para delimitar la idea de filosofía y la propuesta de la fenomenología de Husserl (Dodd 2004, 1) ${ }^{18}$.

La historicidad nos remite al carácter continuo, infinito y teleológico de la tarea correspondiente a la idea de la filosofía, que demanda ser renovada: la historia de la filosofía "[...] posee una unidad espiritual nacida de la unidad y de la fuerza motriz de la tarea, que quiere acceder en el acontecer histórico - en el pensamiento de los que filosofan unos para otros $y$, supratemporalmente, unos con otros- a través de grados diversos de oscuridad, hasta una claridad satisfactoria y, finalmente, hasta la instalación en una

18 "Una meditación histórica retrospectiva del tipo de la que aquí está en juego es, pues, realmente, una automeditación máximamente profunda tendente a alcanzar una autocomprensión sobre lo que en verdad se quiere, una autocomprensión relativa al punto al que se quiere llegar dado lo que se es, en cuanto ser histórico. La automeditación sirve a la decisión, y aquí significa, naturalmente, al mismo tiempo, prosecución de la tarea más propia, de la tarea comprendida y explicada ahora a partir de esa automeditación histórica, la tarea, en fin, que en el presente nos es encomendada en común" (Husserl 1991, § 15, 75-76 [Hua Vl, 73]). 
inteligibilidad plena y perfecta" (Husserl 1991, § 15, 74-75 [Hua Vl, 72-73]). De esta manera, la idea de la filosofía, su sentido, no es uno prefijado ni estático, sino que solo podemos acceder a él a través de la automeditación retrospectiva, dando sentido unitario a sus realizaciones particulares a lo largo de la historia: "[...] la verdad única de una 'consideración teleológica de la historia' de este tipo [...] sólo se muestra en la evidencia de una visión crítica de conjunto que permite vislumbrar tras de los 'hechos históricos' de las teorías filosóficas documentadas y de su aparente contra- y yuxtaposición, una armonía final llena de sentido" (Husserl 1991, § 15, 76 [Hua Vl, 74]). De ahí el avance en zig-zag de la fenomenología (Husserl 1991, § 9, 61 [Hua Vl, 60]).

Cabe resaltar que para Husserl hay tres niveles de historia, los cuales resaltan su vínculo con la filosofía (Hua Vl, 502-503): (1) como unidad de vida espiritual generativa, un "mundo circundante de cosas culturales $\{K u l-$ tur-Sachen-Umwelt\}"; (2) como filosofía, una vida orientada a un propósito particular, que genera una nueva idea de humanidad; y (3) como fenomenología, entendida como meditación sobre la humanidad y su historicidad, y con una función correspondiente. Esto es, la fenomenología trascendental es la forma final de la filosofía - en tanto da el método para una evidencia apodíctica-y, de esta manera, de la humanidad como unidad espiritual de alto nivel (Moran 2012, 219, 223).

\section{§3.4. La tarea de la filosofía como fundamentación última}

Como hemos mencionado, la tarea de la filosofía se desprende de la idea de la filosofía como el imperativo que conlleva para los filósofos: "A la actitud teorética del filósofo corresponde, pues, la decisión constante y previa de dedicar la vida futura, siempre y en el sentido de vida universal, a la tarea de la teoría, a la edificación de conocimiento teórico sobre conocimiento teórico in infinitum" (1991, 342 [Hua VI, 332] ${ }^{19}$. Para Husserl, la tarea de la filosofía abarca las tareas de la ciencia, y, a su vez, la fenomenología es la

19 “Una tarea une a los hombres de modo inextricable: sólo cuando el espíritu retorne a sí mismo desde su orientación ingenua hacia lo exterior y permanezca en sí mismo y puramente en sí mismo, podrá dar razón de sí mismo" (Husserl 1991, 356 [Hua VI, 345-346]). 
efectuación última de la filosofía y de tal tarea (Husserl 1991, 333 [Hua Vl, 322]; cfr. Hua Vl, 494; Husserl 1979, § 64, 230-31 [Hua I, 180]). Su particularidad frente a las otras ciencias consiste en ser una automeditación sobre la posibilidad del conocimiento y no una demostración deductiva de regiones de conocimiento particulares: de ahí su carácter fundante (Husserl 1979, § 64, 230 [Hua l, 180]). Asimismo, se resalta su carácter histórico, el incluir las efectuaciones no actuales de la historia de la filosofía en su horizonte de automeditación permanente (Hua Vl, 488-489).

Entonces, la tarea de la filosofía consiste en la fundamentación última del conocimiento en la evidencia, lo cual equivale, a su vez, a su autorresponsabilidad (Husserl 2013, 466 [Hua V, 139]). La fundamentación está relacionada a la carencia de presupuestos previos, efectuaciones de la subjetividad trascendental sedimentadas y ocultas en su calidad de constituidas (Husserl 2013, 488 [Hua V, 161] $)^{20}$. Husserl sostiene que "[l]a experiencia lisa y llana en la que está dado el mundo de vida es el fundamento último de todo conocimiento objetivo" (1991, § 66, 236 [Hua Vl, 229]). Además, hay una estructura de fundaciones, de forma que cada estrato refiere a uno ulterior, siempre orientados por la unidad de su sentido, la cual no tenemos desde un inicio ni en cada etapa como plenificada, sino como un óptimo al cual tendemos (telos) (Husserl 1991, § 49, 179 [Hua Vl, 173]). Tenemos que pensar tal fundamentación como el motivo o propósito - la tarea- que guía el movimiento del conocimiento, antes que su cimiento fijo (Dodd 2004, 63): “El arché [...] proyectado hacia atrás desde la idea del telos, 'debería' ser la fundación última, absolutamente evidente, no solo del inicio sino de cada etapa de la ciencia" (Rizo-Patrón 2005, 287). Así, el arché o, más bien, su plenificación, es la finalidad a la que tendemos en la realización histórica de la idea de la filosofía y su tarea correspondiente:

20 "Pensar por uno mismo, ser un filósofo autónomo con la voluntad de liberarse de todos los prejuicios, esto exige de uno la elevación a consciencia del hecho de que todo lo que se tiene por obviedades son prejuicios, de que todos los prejuicios son oscuridades provenientes de una sedimentación tradicional y no solamente, pongamos por caso, juicios de verdad incierta, y que esto vale ya para la gran tarea, para la idea que se llama filosofía" (Husserl 1991, $\S 15,75[$ Hua Vl, 73]). 
Una fundación cumplida tan pronto como la tarea ha llegado a la claridad consumada y, con ello, a un método apodíctico tal que cada etapa de la consecución del objetivo es un pasaje permanente para nuevas etapas que tienen el carácter de éxitos absolutos, esto es, de lo apodíctico. La filosofía como tarea infinita habría llegado así a su comienzo apodíctico, a su horizonte apodíctico de prosecución (Husserl 1991, § 15, 76 [Hua Vl, 73]).

Se trata de una tarea infinita, compuesta, a su vez, por una infinidad de tareas desplegada en la historia y vulnerable a correcciones, mas, aun así, realizable (Husserl 2013, 466 [Hua V, 139]): “\{el a priori\} sólo puede ser mostrado en una relatividad, en un despliegue de horizontes", y en la misma dación de nuevos escorzos notamos "limitaciones pasadas por alto y algunos horizontes no percibidos", los cuales "instan al cuestionamiento de nuevas correlaciones que están ligadas de forma inquebrantable con las ya mostradas" (Husserl 1991, § 46, 168 [Hua Vl, 162]). Como mencionamos, el vínculo con la historia también se remonta a la génesis histórica de la filosofía en la que se traza su tarea (Husserl 2013, 489 [Hua V, 162]). Aquí, cabe distinguir la filosofía como factum histórico de su idea en tanto tarea infinita: "La filosofía históricamente efectiva en cada caso es el intento más o menos conseguido de realizar la idea rectora de la infinitud y, con ella, la totalidad incluso de las verdades"; esto es, ante la posibilidad de errores y necesidad de correcciones, "[h]ay que contar con la constante amenaza de recaer en unilateralidades y en satisfacciones demasiado apresuradas, que se vengan en las contradicciones sobrevinientes" (Husserl 1991, 348 [Hua VI, 338]). De ahí que:

Solamente en la fundación final se revela esto <la unidad intencional de la tarea de la filosofía>, sólo a partir de ella puede manifestarse la orientación unitaria de todas las filosofías y filósofos, y sólo a partir de ella puede lograrse una claridad a cuya luz resultan comprensibles los pensadores como jamás ellos mismos hubieran podido comprenderse (Husserl 1991, § 15, 76 [Hua Vl, 74]).

En la captación del valor del conocimiento y la consecuente tendencia hacia él por parte del filósofo - esto es, desde el aspecto noético- la falsedad "[...] sólo queda excluida, y la pretensión de apodicticidad sólo queda justificada, cuando se llega a una fundamentación mediata y absolutamente apodíctica que la reenvía a esa única evidencia primigenia 
absoluta a la que todo conocimiento científico debe ser conducido - si es que una filosofía tiene que ser posible" (Husserl 1991, § 17, 82 [Hua Vl, 80]). Correlativamente, desde el aspecto noemático, "[l]a verdad ideal se convierte así en un valor absoluto, que trae consigo una transformación universal de la praxis", ya que, "si la idea general de la verdad en sí se convierte en norma universal de todas las verdades relativas [...], entonces esto afecta, obviamente, también, a todas las normas tradicionales, a las del derecho, a las de la belleza, a las de la conveniencia, a las de los valores personales dominantes, a las de los valores de los caracteres personales, etc." (Husserl 1991, 343 [Hua Vl, 333-334]). Aquí se muestra el vínculo entre lo teorético y lo práctico, enfatizándose la articulación de los aspectos de la razón que detalla Husserl con especial énfasis en esta obra y que, como mencionamos, sirve de presupuesto de sus ideas. Recordemos que el papel de lo emotivo-valorativo destaca en tanto según el orden de fundación de los actos, todo acto práctico se funda en la captación de un valor (Husserl 2013, §116, 362 [Hua III/1, 239]). Es decir, provee su motivación y criterio normativo.

\section{$\S 4$. Conclusiones}

Podemos establecer aquí, en esta proyección al infinito, la relación de esta consideración de la tarea de la filosofía, vinculada a la fundamentación del conocimiento —últimamente, la autorresponsabilidad del filósofo- y el tercer sentido de evidencia y delimitación entre lo inmanente y trascendente, en tanto no se reduce a lo dado actual, sino que se extiende a lo inactual pasado y posible, incluyendo las experiencias ajenas, a las cuales no tenemos acceso en primera persona (Hua Vl, 494). Asimismo, como mencionamos, resalta que en el desarrollo de este último sentido hay una etapa estática — correspondiente a Ideas I- y una genética — plasmada en la Crisis-, en la cual adquiere un sentido más profundo debido al reconocimiento del carácter dinámico, temporal e histórico que atraviesa y estructura la intencionalidad.

El despliegue de la tarea de la filosofía es, entonces, el despliegue de la razón, entendido como la dinámica teleológico-normativa de la 
intencionalidad: la filosofía"es racionalismo que se diferencia en sí según los distintos niveles del movimiento de intención y cumplimiento; es la ratio en el constante movimiento de la autoaclaración, comenzada por la primera irrupción de la filosofía en la humanidad, cuya razón innata todavía estaba con anterioridad totalmente en el estadio de la cerrazón, de la oscuridad nocturna" (Husserl 1991, § 73, 281 [Hua VI, 273]). Por lo tanto, al elevarla al nivel intersubjetivo y tomar en cuenta la historicidad, la filosofía ha de ser entendida "[...] como comunidad profesional de los filósofos que se extiende, y como movimiento comunitario de formación que se coextiende con aquéIla" (Husserl 1991, 342-343 [Hua Vl, 333]). Asimismo, como adelantamos, posee una doble influencia espiritual en la humanidad: teórico-crítica y, consecuentemente, práctica, cuando los intereses mundano-prácticos y teoréticos son sintetizados (Husserl 1991, 343-344 [Hua VI, 333]).

Si la tarea de la filosofía plantea la responsabilidad máxima del filósofo para con la humanidad, es debido a que solo podemos tematizarla en su Selbstbesinnung (Husserl 1991, 349 [Hua Vl, 338]). Como mencionamos, esto se relaciona a la concepción de la razón en Husserl, como una unidad teleológicamente articulada, cuyo mejor retrato se encuentra hacia el final de la obra ${ }^{21}$. Entonces, el concepto de responsabilidad en la Crisis se establece como producto de la automeditación (Selbstbesinnung) ejercida por el filósofo, correspondiente a la tarea implicada en la idea de la filosofía. Esta, en tanto búsqueda de la fundamentación última, ha de proveer un método para alcanzar la evidencia apodíctica, donde se expresa la modificación de la delimitación de los ámbitos inmanente y trascendente, para describir cabalmente el tipo de evidencia que justifica las afirmaciones y

21 Husserl describe la labor del filósofo que lleva a cabo la meditación fenomenológica señalando que: "[...] lo hace comprendiéndose como racional y comprendiendo que la razón es racional en el querer-ser-racional, que esto significa una infinitud de la vida y del esforzarse hacia la razón, que la razón indica precisamente aquello hacia lo que el hombre en tanto que hombre desea llegar en su máxima intimidad, aquello que únicamente puede satisfacerlo, hacerlo 'bienaventurado', que la razón no admite ninguna distinción en 'teórica', 'práctica' y 'estética' y ello por más que se haga, que el ser-hombre es un ser-teleológico y un deber-ser y que esta teleología impera en todos y cada uno de los haceres y proyectos yoicos, que en todos estos haceres y proyectos puede comprender el telos apodíctico mediante la autocomprensión y que este conocimiento de la autocomprensión última no tiene otra configuración que la de la autocomprensión según principios aprióricos, que la de la autocomprensión en la forma de filosofía" (Husserl 1991, § 73, 283 [Hua Vl, 275]). 
constituciones de sentido, lo cual trasciende al ámbito exclusivo de la ciencia hacia la ética (Rizo-Patrón 2005, 291). La fenomenología, concebida por Husserl como filosofía primera, es un ejercicio de automeditación antes que un análisis exterior, con pretensiones puramente objetivas, como se había privilegiado en la Modernidad.

Dicha fenomenología supera el objetivismo naturalista y, en general, todo objetivismo, del único modo posible, a saber, haciendo que el que filosofa parta de su yo y, en concreto, de su yo en cuanto puro productor de todas sus valideces, de las que se convierte en espectador puramente teórico. En esta actitud, y desde ella, resulta posible elaborar una ciencia del espíritu absolutamente autónoma, en la forma de una autocomprensión consecuente y de una comprensión del mundo en tanto que rendimiento del espíritu (Husserl 1991, 356 [Hua Vl, 356]).

Recibido: 17/04/2020

Aceptado: 06/06/2020

\section{Bibliografía}

Bernet, Rudolf, 2004. Husserl's Transcendental Idealism Revisited. Interdisziplinäre Phänomenologie 1, 117-140.

Chu, Mariana, 2013. La objetividad de los valores en Husserl y Scheler. Una "disputa fenomenológica". Investigaciones fenomenológicas 4 (2), 279-293.

Dodd, James, 2004. Crisis and Reflection. An Essay on Husserl's Crisis of European Sciences. Dordrecht: Kluwer Academic Publishers.

Hart, James G., 1992. The Person and the Common Life. Studies in a Husserlian Social Ethics. Dordrecht: Kluwer Academic Publishers.

Husserl, Edmund, 1950-2015. Husserliana: Edmund Husserl - Gesammelte Werke, 43 volúmenes (Hua I-XLIII). La Haya, Dordrecht, Nueva York: Martinus Nijhoff, Kluwer Academic Publishers y Springer. Se cita en el cuerpo del artículo los tomos de esta colección con la sigla Hua seguida por el número de volumen escrito en cifras romanas.

- Hua I, 1973. Cartesianische Meditationen und Pariser Vorträge. Editado por Stephan Strasser. La Haya: Martinus Nijhoff.

- 1979. Meditaciones Cartesianas. Traducción de Mario Presas. Madrid: Ediciones Paulinas. 
- Hua III/1, 1976. Ideen zu einer reinen Phänomenologie und phänomenologische Philosophie. Erstes Buch: Allgemeine Einführung in die reine Phänomenologie. Editado por Karl Schuhmann. La Haya: Martinus Nijhoff.

- 2013. Ideas relativas a una fenomenología pura y una filosofía fenomenológica. Libro primero: Introducción general a la fenomenología. Traducción de Antonio Zirión Quijano. México D.F.: Fondo de Cultura Económica, Universidad Autónoma de México.

- Hua IV, 1952. Ideen zu einer reinen Phänomenologie und phänomenologische Philosophie. Zweites Buch: Phänomenologische Untersuchungen zur Konstitution. Editado por Marly Biemel. La Haya: Martinus Nijhoff.

- 2005. Ideas relativas a una fenomenología pura y una filosofía fenomenológica. Libro Segundo: Investigaciones fenomenológicas sobre la constitución. Traducción de Antonio Zirión Quijano. México D.F.: Fondo de Cultura Económica, Universidad Nacional Autónoma de México.

- Hua V, 1952. Ideen zu einer reinen Phänomenologie und phänomenologischen Philosophie. Drittes Buch:Die Phänomenologie und die Fundamente der Wissenschaft. Editado por Marly Biemel. La Haya: Martinus Nijhoff.

- Hua VI, 1954. Die Krisis der europäischen Wissenschaften und die transzendentale Phänomenologie. Eine Einleitung in die phänomenologische Philosophie. Editado por Walter Biemel. La Haya: Martinus Nijhoff.

- 1991. La crisis de las ciencias europeas y la fenomenología trascendental. Traducción de Jacobo Muñoz y Salvador Mas. Barcelona: Crítica.

- Hua XI, 1966. Analysen zur passiven Synthesis. Aus Vorlesungs- und Forschungsmanuskripten, 1918-1926. Editado por Margot Fleischer. La Haya: Martinus Nijhoff.

- Hua XIV, 1973. Zur Phänomenologie der Intersubjektivität. Texte aus dem Nachlass. Zweiter Teil: 1921-1928. Editado por Iso Kern. La Haya: Martinus Nijhoff.

- Hua XV, 1973. Zur Phänomenologie der Intersubjektivität. Texte aus dem Nachlass. Dritter Teil: 1929-1935. Editado por Iso Kern. La Haya: Martinus Nijhoff.

- Hua XVIII, 1975. Logische Untersuchungen. Erster Band. Prolegomena zur reinen Logik. Editado por Elmar Holenstein. La Haya: Martinus Nijhoff.

- 2006. Investigaciones lógicas I. Traducción de Manuel G. Morente y José Gaos. Madrid: Alianza.

- Hua XIX/1, 1984. Logische Untersuchungen. Zweiter Band - Erster Teil: Untersuchungen zur Phänomenologie und Theorie der Erkenntnis. Editado por Ursula Panzer. La Haya: Martinus Nijhoff.

- Hua XIX/2, 1984. Logische Untersuchungen. Zweiter Band - Zweiter Teil: Untersuchungen zur Phäomenologie und Theorie der Erkenntnis. Editado por Ursula Panzer. La Haya: Martinus Nijhoff. 
- 1929. Investigaciones lógicas. Traducción de José Gaos y Manuel García Morente. Madrid: Revista de Occidente.

- Hua XXVII, 1989. Aufsätze und Vorträge (1922-1937). Editado por Hans Rainer Sepp y Thomas Nenon. Dordrecht: Kluwer Academic Publishers.

- Hua XXVIII, 1988. Vorlesungen über Ethik und Wertlehre (1908-1914). Editado por Ullrich Melle. Dordrecht: Kluwer Academic Publishers.

- 2002. Renovación del hombre y de la cultura. Traducción de Agustín Serrano de Haro. Barcelona: Anthropos.

- Hua XXXVII, 2004. Einleitung in die Ethik: Vorlesungen Sommersemester 1920/1924. Editado por Henning Peucker. Dordrecht: Kluwer Academic Publishers.

- Hua XLII, 2014. Grenzprobleme der Phänomenologie: Analysen des Unbewusstseins und der Instinkte. Metaphysik. Späte Ethik (Texte aus dem Nachlass 1908-1937). Editado por Rochus Sowa y Thomas Vongehr. Dordrecht: Springer.

Husserl, Edmund, 1980. Experiencia y juicio. Traducción de Jas Reuter. México D.F.: Universidad Nacional Autónoma de México.

Moran, Dermot, 2012. Husserl's Crisis of the European Sciences and Transcendental Phenomenology: An Introduction. Cambridge: Cambridge University Press.

Quepons, Ignacio, 2016. Vida afectiva y conciencia de valor: observaciones sobre la génesis constitutiva de la objetividad axiológica en la fenomenología de Husserl. Revista del Centro de Investigación de la Universidad La Salle 12 (46), 103-128.

Rizo-Patrón, Rosemary, 2005. La situación del filósofo que comienza: dilemas husserlianos en torno a la primera evidencia. Acta fenomenológica latinoamericana 2. Lima: Círculo Latinoamericano de Fenomenología, Pontificia Universidad Católica del Perú, 273-292.

- 2015. El exilio del sujeto. Bogotá, Lima: Aula de Humanidades, Fondo Editorial de la Pontifica Universidad Católica del Perú.

San Martín, Javier, 2008. La fenomenología de Husserl como utopía de la razón. Madrid: Biblioteca Nueva.

Schuhmann, Karl. 1981. Husserl-Chronik. Denk-und Lebensweg Edmund Husserls. The Hague: Martinus Nijhoff Publishers.

Steinbock, Anthony, 2019. Participation and Critique: Discernment of the Heart. Conferencia presentada en las XV Jornadas Peruanas de Fenomenología y Hermenéutica. Pontificia Universidad Católica del Perú. Lima, 22 de noviembre de 2019.

Walton, Roberto, 2017. Horizonality and Legitimation in Perception, Affectivity and Volition. En: Perception, Affectivity, and Volition in Husserl's Phenomenology, eds. Roberto Walton y otros. Cham: Springer, 3-20.

Walton, Roberto y otros (eds.), 2017. Perception, Affectivity, and Volition in Husserl's Phenomenology. Cham: Springer. 